\title{
Effects of applying stress on the electron field emission properties in amorphous carbon thin films
}

\author{
C. H. P. Poa and S. R. P. Silva ${ }^{a)}$ \\ Advanced Technology Institute, School of Electronics and Physical Sciences, University of Surrey, \\ Guildford, Surrey, GU2 7XH, United Kingdom \\ R. G. Lacerda, G. A. J. Amaratunga, and W. I. Milne \\ Engineering Department, University of Cambridge, Trumpington Street, Cambridge, CB2 1PZ, \\ United Kingdom \\ F. C. Marques \\ Universidade Estadual de Campinas, Unicamp, Instituo de Fisica "Gleb Wataghin"-13083-970, \\ Campinas-SP, Brazil
}

(Received 29 December 2004; accepted 22 April 2005; published online 1 June 2005)

\begin{abstract}
Diamond-like carbon (DLC) films have always had high intrinsic stress due to their metastable structure and the fine balance between film density and bond stability. We show the effects of high intrinsic stress on the electron field emission performance, where a lower electric field for emission is recorded with increasing stress in the DLC films. In addition to examining "as deposited" films with different magnitudes of intrinsic stress, we subject the DLC films to external pressure by physically bending the $a-\mathrm{C} /$ silicon substrates. The result is a phenomenon where electrons are "squeezed" out of the films, and can be applied to the fabrication of stress sensors. (C) 2005 American Institute of Physics. [DOI: 10.1063/1.1940129]
\end{abstract}

Amorphous carbon $(a-C)$ thin film research has attracted enormous interest for the last two decades due to its unique properties $^{1}$ and possible application as cold cathodes for electron field emission. ${ }^{2}$ However, the ability of $a-C$ thin films to possess high intrinsic stress has been undesirable, particularly in view of microelectromechanical system applications. In fact, $a$-C films with intrinsic stress values $(\sim 12 \mathrm{GPa})$ of almost one order of magnitude higher than those found in other amorphous materials $(<1 \mathrm{GPa})$ such as $a-\mathrm{Si}, a-\mathrm{Ge}$, or metals have been reported. Excessive stress on thin films can cause adhesion problems where the film delaminates from the substrate. The presence of high stress on $a$-C has been reported to have induced changes in the electronic properties such as electrical conductivity. ${ }^{3}$ The a-C films with high $s p^{2}$ concentrations $(\sim 90 \%)$ can have high intrinsic stress and a local density close to those found in tetrahedral amorphous carbon (TAC) thin films. ${ }^{1}$ The high compressive stress in these graphite-like $a$-C (GAC) films is a result of the ion bombardment during the deposition process that "knock-on" carbon which subimplant into the films and thus create regions of high stress. ${ }^{4}$ This results in the densification of the films which increase the local density. It is known from theoretical studies that by applying pressure (stress) to a material one can modify its electronics properties, e.g., band structure, resistivity, position of Fermi level. ${ }^{5,6}$ The influence of internal pressure on the electron emission properties of nanocomposite thin films which contain nano-graphite has been reported by Poa et al. $^{7}$ in which by varying the deposition conditions, the properties of the nano-graphite as a function of stress were studied. The observed low threshold field was explained by the amount of intrinsic stress induced within the carbon matrix. Basically, the variation in field emission characteristics with intrinsic

\footnotetext{
a) Author to whom correspondence should be addressed; electronic mail:
} s.silva@surrey.ac.uk stress can be driven by two effects: (1) band structure modifications in the film and (2) increases in local density within the film. In both cases, the beneficial effect is the lowering of field emission threshold fields and/or increasing the electric field enhancement factor. Due to the dielectric mismatch within the carbon films, electric field lines terminate at the more conductive regions of the film surface. The crowding of the field lines result in an increase in the local electric field between the dielectric interfaces, increasing the field enhancement for electron emission.

In this work the $a-\mathrm{C} /$ silicon substrate is physically bent to induce external stress on the $a$-C films. The effect of the applied external pressure was then examined in terms of the electron field emission characteristics. We further show that previous results attributed purely to the effects of $s p^{2} / s p^{3}$ bond hybridizations may equally well be explained using an argument based on stress in thin films.

The graphitic $a-\mathrm{C}$ (GAC) films studied in this investigation were deposited by an ion beam assisted deposition (IBAD) technique using two Kauffman ion sources. Films produced from this technique are mainly graphitic, with a $s p^{2}$ concentration of up to $90 \%$ as determined by electron energy loss spectroscopy. Details of the deposition system have been described elsewhere. ${ }^{8}$ Stress measurements were performed using films deposited on $c$-Si bars of $20 \mathrm{~mm}$ by $5 \mathrm{~mm}$, and applying an optical bending beam method. ${ }^{9}$ The field emission (FE) characteristics of these films were determined using a sphere-to-plane technique with a separation distance between the anode and cathode of $20 \mu \mathrm{m}$. The threshold field $\left(E_{\mathrm{th}}\right)$ is defined as the macroscopic electric field where an emission current of $1 \mathrm{nA}$ is observed. The samples are bent using a fiber placed in the middle of the sample, with two glass slides used to clip down both ends of the sample to create a curvature as shown in Fig. 1. Different sizes of fibers are used, from 50 to $250 \mu \mathrm{m}$, which allows for different compressive stresses to be applied onto the $a$-C films. 


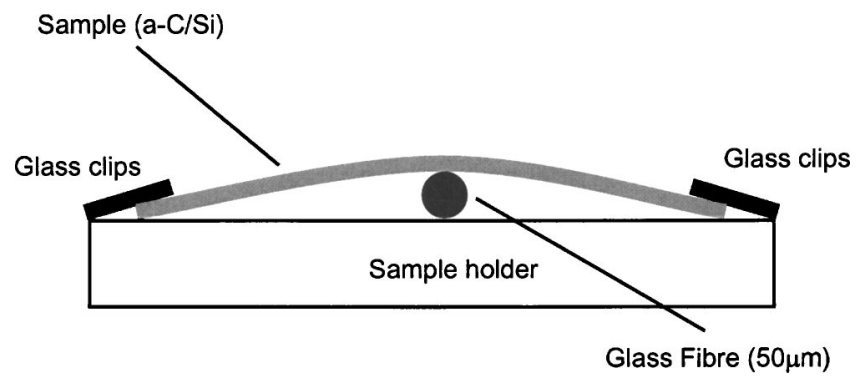

FIG. 1. The experimental setup for the 3 point bending stage.

Amorphous carbon films with a high degree of $s p^{3}$ bonding, known as tetrahedral $a$-C (TAC), have been shown to be good electron emitters, Fig. 2. Previous examination of the field emission properties of TAC have been explained by considering the influence of different bond hybridization, with higher $s p^{3}$ bonding found to enhance field emission. ${ }^{10}$ We take this data and now plot it as a function of ion energy with the data found in the literature together with data from our own highly $s p^{2}$-rich $a$-C films, Fig. 2 . The effects of intrinsic stress and ion energy for both TAC and GAC are shown in Fig. 2(a). The TAC films were deposited using the filtered ion beam technique by Fallon et al. ${ }^{11}$ and the intrinsic stress in Fig. 2 is seen to be maximum (11 GPa) at about 140 $\mathrm{eV}$ ion energy. The GAC films deposited using IBAD technique with krypton show a maximum stress of $11.7 \mathrm{GPa}$ at $300 \mathrm{eV}$. This optimum energy is dependent on many factors with the deposition system being used primary among them. ${ }^{12}$ Despite two different deposition techniques, similar trends have been observed, where a maximum stress is observed at an optimum energy, which starts to decrease with increasing energy.

The field emission results obtained from Ref. 10, are shown in Fig. 2(b) for the TAC and GAC films (this work), where the lowest threshold fields $(8$ and $23 \mathrm{~V} / \mu \mathrm{m}$, respectively) are observed in the region where the films have the highest stress. The variation of threshold field and intrinsic stress for all the $a$-C films, in a combined plot, is shown in Fig. 3, where it can be seen clearly that the lowest threshold fields are from those films with the highest intrinsic stress. It is important to note that the definition of field emission threshold field in Ref. 10 is different from this work where an emission current density of $1 \mu \mathrm{A} / \mathrm{cm}^{2}$ was used to define the threshold field. However, it is still suitable to compare the data qualitatively as the threshold fields are consistent with the results reported in the literature.

Satyanarayana et al. ${ }^{10}$ proposed that for TAC films to exhibit low threshold fields, high $s p^{3}$ content within the films should be present. It was suggested by the authors that, negative electron affinity may be responsible for the reduction of the emission barrier. However, TAC is not a negative electron affinity material with its work function being 3.5-4 $\mathrm{eV}^{13}$ Interestingly, the threshold fields decrease with increasing $s p^{3}$ concentration, which is the opposite dependence to laser ablated TAC films ${ }^{14}$ and GAC films shown in Fig. 3. The samples studied by us are highly graphitic and have the lowest emission threshold fields when the $s p^{3}$ content was a minimum. Therefore, this suggests that other mechanisms are important in controlling the field emission properties and in some cases more dominant than the $s p^{3}$ bond content.

To investigate the effects of externally applied stress on the field emission from carbon thin films, three films with Downloaded 30 Mar 2009 to 131.227.178.132. Redistribution subje

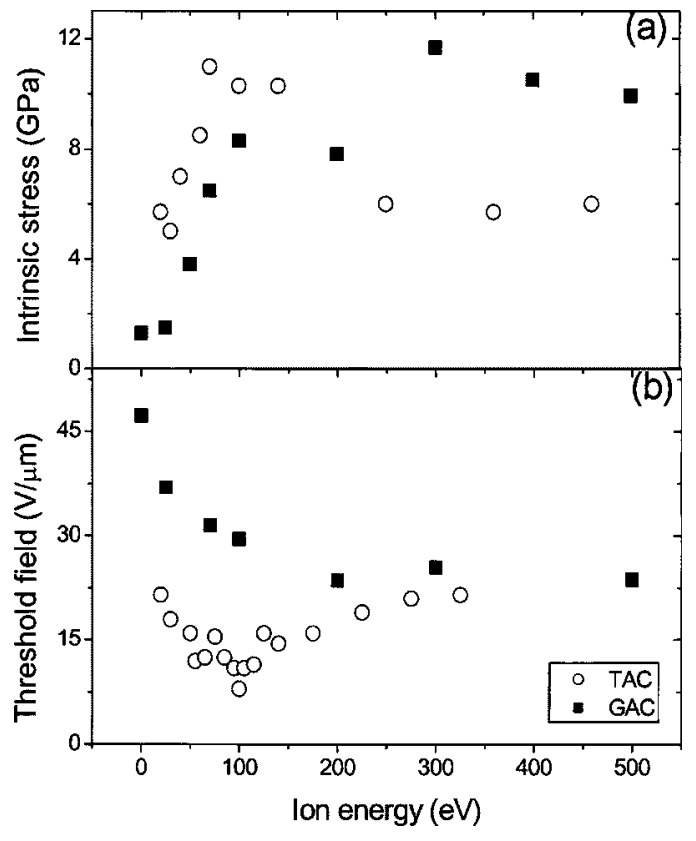

FIG. 2. The variation of intrinsic stress as a function of ion energy for (a) TAC films and GAC films. The variation of field emission threshold field as a function of ion energy for (b) TAC films and GAC films.

different stress are subjected to bending experiments to stimulate controllable external stress application on films. Different krypton assisted films are used to represent films with low (1 GPa), medium (6.5 GPa) and high intrinsic stress $(11.7 \mathrm{GPa})$. By bending the substrate as shown in Fig. 1, the change in curvature results in an increase of the stress within the thin film. The stress is compressive on the Si/GAC interface and tensile on the GAC/air interface. The changes in the substrate curvature due to bending are calculated using Stoney's equation. It is difficult to calculate the induced stress within the thin GAC films and therefore the curvature in Fig. 1 is used on a qualitative basis for comparison to curvature modifications. The bending of the low stress film shows a very interesting sequence of results, as shown in Fig. 4. Upon the application of external stress, $E_{\text {th }}$ initially at $37 \mathrm{~V} / \mu \mathrm{m}$ falls to $24 \mathrm{~V} / \mu \mathrm{m}$, as the amount of stress is increased due the change in the curvature. This reduction of $E_{\mathrm{th}}$ we believe is due to the increase of internal stress by having an external compressive force applied on the film. As the curvature is further increased to the highest level, we believe there is significant structural modification (reversible) in the graphitic planes which relaxes the microstructure due to the

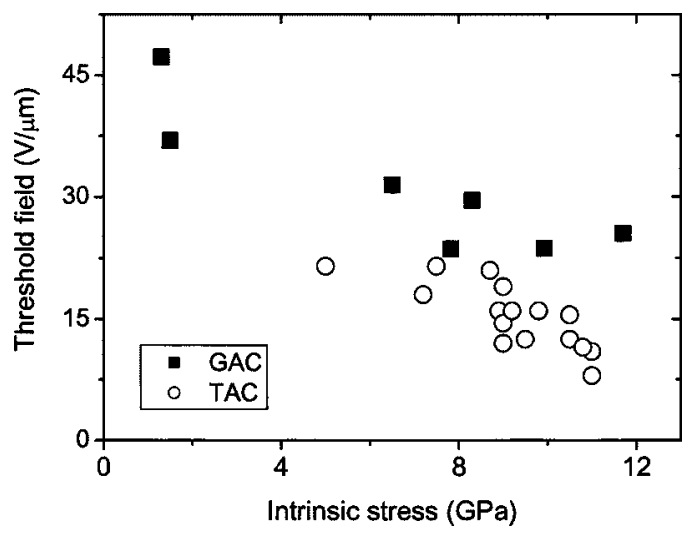

FIG. 3. Variation of $E_{\text {th }}$ against intrinsic stress for both TAC and GAC films. to AIP license or copyright; see http://apl.aip.org/apl/copyright.jsp 


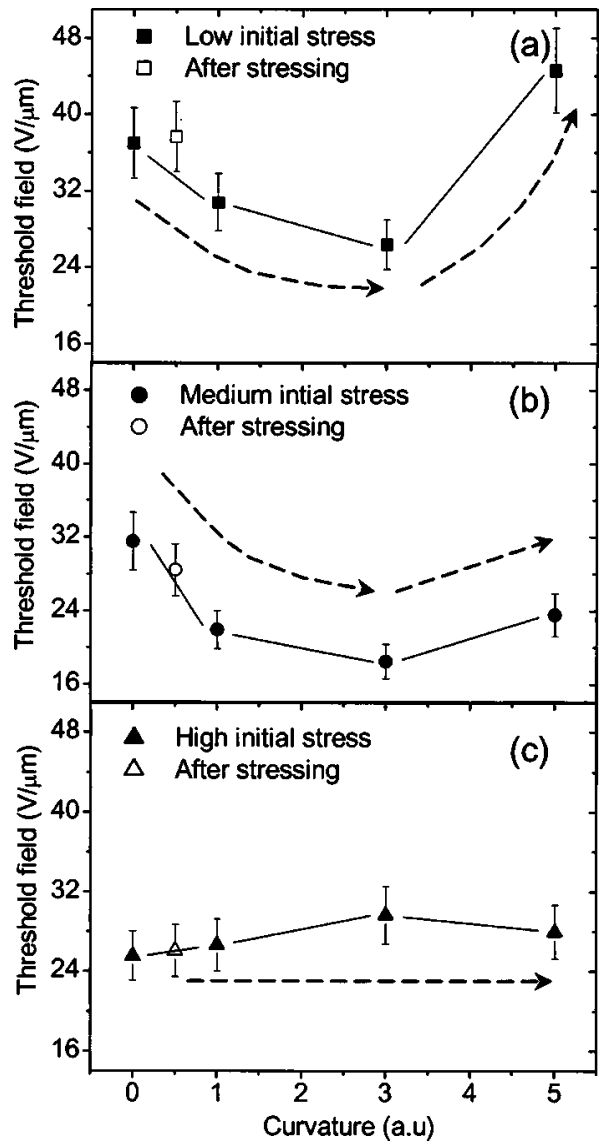

FIG. 4. Films with initial stress of low (1.5 GPa), medium (5 GPa) and high $(12 \mathrm{GPa}$ ) are bent with fibers of $50 \mu \mathrm{m}$ (curvature 1), $125 \mu \mathrm{m}$ (curvature 3) and $250 \mu \mathrm{m}$ (curvature 5). Variation of $E_{\mathrm{th}}$ are plotted against the curvature induced by the fiber thickness.

$s p^{2}$ planes slipping relative to each other. Hence, $E_{\text {th }}$ increases to $44 \mathrm{~V} / \mu \mathrm{m}$ at a maximum stress. Interestingly, when the sample is tested after the curvature has been removed, the field emission threshold field resets itself to the original value of $35 \mathrm{~V} / \mu \mathrm{m}$, emphasizing the elastic behavior of the film which can be subjected to repeated bending cycles. This appears to support a phenomenon which is more likely to be related to electronic modification as opposed to structural changes. In the case of the films with medium stress, similar trends are observed in the bending process. However, when the film with the highest stress is bent, there is no decrease of $E_{\text {th }}$ below the initial value. This can be attributed by the high intrinsic stress within the films not being exceeded by the external pressure on the film or the phenomenon responsible for the electron density/decrease in threshold exceeding the "stress threshold value."

The effect of stress on the field emission properties of $a$-C thin films has not be often discussed in the literature. Commonly, intrinsic stress is only associated with the $s p^{3}$ concentration in $a$-C films and is ignored due to delamination of the films. However, from the bending results in Fig. 4, it is clear that stress can play a crucial role in the field emission results. Interestingly, the threshold field resets itself after the bending experiment which suggests that the modification due to stress is reversible. In comparison to single wall car- bon nanotubes, applying radial deformation to a nanotube can vary the band gap from semiconducting to metallic. ${ }^{15}$ Depending on the type of tubes and the relative position of these bands, the band gap displays different behavior to strain. In general, the band gap decreases and eventually closes to form a semiconducting to metallic transition. However, the band structure in amorphous materials is not well defined, for example, in $a$-C films, it is predominantly controlled by its $\pi$ and $\sigma$ bonding and there are localized states found in the midgap region. It is possible that under strain, the localized states within the $a$-C film are pushed closer together and hence deform the band structure to favor electron emission.

This experiment illustrates a drop of $10-15 \mathrm{~V} / \mu \mathrm{m}$ by simply creating a curvature of 3 on the film with low and medium stress. The fact that the process is reversible and highly reproducible bodes well for the possibility of fabrication of highly sensitive pressure sensor based on electron field emission. For example, for a constant electric field of $35 \mathrm{~V} / \mu \mathrm{m}$ a FE current of $1 \mathrm{nA}$ is recorded. Now, when the curvature of 3 is applied to the film, while the constant electric field of $15 \mathrm{~V} / \mu \mathrm{m}$ is applied, a new current of $1 \mu \mathrm{A}$ is detected. Due to a small change in threshold field, very large changes in FE currents are recorded.

In conclusion, we have examined the variation of electron field emission with stress. The effect of intrinsic stress is shown to make a significant contribution to factors controlling the process of electron field emission. The lowest threshold fields were observed in films possessing the highest stress. By bending the films, we observe variations in the threshold field which we believe to be the result of electron density variations within these films.

All authors gratefully acknowledge financial support from the EPSRC Carbon Based Electronics program.

\footnotetext{
${ }^{1}$ See, for example, Properties of Amorphous Carbon, edited by S. R. P. Silva (INSPEC, IEE, London, 2003).

${ }^{2}$ G. A. J. Amaratunga and S. R. P. Silva, Appl. Phys. Lett. 68, 2529 (1996).

${ }^{3}$ R. G. Lacerda, P. Hammer, C. M. Lepienski, F. Alvarez, and F. C. Marques, J. Vac. Sci. Technol. A 19, 971 (2001).

${ }^{4}$ J. Schwan, S. Ulrich, H. Roth, H. Ehrhardt, S. R. P. Silva, J. Robertson, R. Samlenski, and R. Brenn, J. Appl. Phys. 79, 1416 (1996).

${ }^{5}$ C. Kilic, H. Mehrez, and S. Ciraci, Phys. Rev. B 58, 7872 (1998).

${ }^{6}$ C. Uher, R. L. Hockey, and E. Ben-Jacob, Phys. Rev. B 35, 4483 (1987).

${ }^{7}$ C. H. Poa, R. G. Lacerda, D. C. Cox, S. R. P. Silva, and F. C. Marques, Appl. Phys. Lett. 81, 853 (2002).

${ }^{8}$ F. Alvarez, M. C. dos Santos, and P. Hammer, Appl. Phys. Lett. 73, 3521 (1998).

${ }^{9}$ R. G. Lacerda, P. Hammer, C. M. Lepienski, F. Alvarez, and F. C. Marques, J. Vac. Sci. Technol. A 19, 971 (2001).

${ }^{10}$ B. S. Satyanarayana, A. Hart, W. I. Milne, and J. Robertson, Appl. Phys. Lett. 71, 1430 (1997).

${ }^{11}$ P. J. Fallon, V. S. Veerasamy, C. A. Davis, J. Robertson, G. A. J. Amaratunga, W. I. Milne, and J. Koskinen, Phys. Rev. B 48, 4777 (1993).

${ }^{12}$ J. Schwan, S. Ulrich, T. Theel, H. Roth, H. Ehrhardt, P. Becker, and S. R. P. Silva J. Appl. Phys. 82, 6024 (1997).

${ }^{13}$ J. Robertson, J. Vac. Sci. Technol. B 17, 659 (1999).

${ }^{14}$ N. Missert, T. A. Friedmann, J. P. Sullivan, and R. G. Copeland, Appl. Phys. Lett. 70, 1995 (1997).

${ }^{15}$ O. Gülseren, T. Yildirim, S. Ciraci, and Ç. Klç, Phys. Rev. B 65, 155410 (2002).
} 